\title{
GENERALIZED MARCINKIEWICZ-ZYGMUND TYPE INEQUALITIES FOR RANDOM VARIABLES AND APPLICATIONS
}

\author{
Pingyan Chen And Soo HaK Sung
}

\begin{abstract}
It is shown that if the higher order Marcinkiewicz-Zygmund type inequality holds, then some generalized Marcinkiewicz-Zygmund type inequality holds, in particular, the lower order Marcinkiewicz-Zygmund type inequality also holds. No additional assumptions are made on the random variables. As applications, a generalized $C_{r}$-inequality and a weak law of large numbers for pairwise independent random variables are obtained.
\end{abstract}

Mathematics subject classification (2010): 60F15.

Keywords and phrases: Marcinkiewicz-Zygmund inequality, moment inequality, $C_{r}$-inequality, weak law of large numbers.

\section{REFERENCES}

[1] N. Asadian, V. Fakoor, A. Bozorgnia, Rosenthal's type inequalities for negatively orthant dependent random variables, J. Iran. Statist. Soc. 5 (2006), 69-75.

[2] B. VON BAHR, C. G. ESSEEN, Inequalities for the $r$ th absolute moment of a sum of random variables, $1 \leqslant r \leqslant 2$, Ann. Math. Statist. 36 (1965), 299-303.

[3] D. L. Burkholder, Martingale transforms, Ann Math. Statist. 37 (1966), 1494-1504.

[4] T. K. CHANDRA, Uniform integrability in the Cesàro sense and the weak law of large numbers, Sankhyā Ser. A 51 (1989), 309-317.

[5] P. Chen, P. BAI, S. H. Sung, The von Bahr-Esseen moment inequality for pairwise independent random variables and applications, J. Math. Anal. Appl. 419 (2014), 1290-1302.

[6] M. HADJIKYRIAKOU, Marcinkiewicz-Zygmund inequality for nonnegative $N$-demimartingales and related results, Statist. Probab. Lett. 81 (2011), 678-684.

[7] E. L. Lehmann, Some concepts of dependence, Ann. Math. Statist. 37 (1966), 1137-1153.

[8] P. MATULA, A note on the almost sure convergence of sums of negatively dependent random variables, Stat. Probab. Lett. 15 (1992), 209-213.

[9] F. MóRICZ, Moment inequalities and the strong laws of large numbers, Z. Wahrsch. Verw. Gebiete 35 (1976), 299-314.

[10] Q. M. SHAO, A moment inequality and its applications, Acta Math. Sinica (Chinese Series) 31 (1988), 736-747.

[11] Q. M. Shao, Maximal inequalities for partial sums of $\rho$-mixing sequences, Ann. Probab. 23 (1995), 948-965.

[12] Q. M. SHAO, A comparison theorem on moment inequalities between negatively associated and independent random variables, J. Theoret. Probab. 13 (2000), 343-356.

[13] S. Utev, M. Peligrad, Maximal inequalities and an invariance principle for a class of weakly dependent random variables, J. Theoret. Probab. 16 (2003), 101-115.

[14] S. C. YANG, Almost sure convergence of weighted sums of mixing sequences, J. Sys. Sci. Math. Sci. 15 (1995), 254-265.

[15] L. X. ZHANG, A functional central limit theorem for asymptotically negatively dependent random fields, Acta Math. Hungar 86 (2000), 237-259. 